\title{
The Formation of Excimers in Isoquinolinyl Pyrazolate Pt(II) Complexes: Role of Cooperativity Effects
}

\author{
Chattarika Sukpattanacharoen, ${ }^{a, b}$ Prashant Kumar, ${ }^{a}$ Yun Chi, ${ }^{c}$ \\ Nawee Kungwan ${ }^{b, d}$, Daniel Escudero, ${ }^{a} *$ \\ ${ }^{a}$ Department of Chemistry, KU Leuven, Celestijnenlaan 200F, B-3001 Leuven, Belgium \\ ${ }^{b}$ Department of Chemistry, Faculty of Science and Graduate School, Chiang Mai University, \\ Chiang Mai, 50200, Thailand \\ ${ }^{c}$ Department of Materials Science and Engineering and Department of Chemistry, City \\ University of Hong Kong, Kowloon Tong, Hong Kong SAR \\ ${ }^{d}$ Center of Excellence in Material Science and Technology, Chiang Mai University, \\ 50200, Thailand
}

KEYWORDS. Isoquinolinyl pyrazolate Pt(II) complexes; Photophysics; Aggregates; Photoluminescence, Cooperativity between non-covalent interactions; Excimers; Energy decomposition analysis, DFT calculations 
ABSTRACT. The interplay between non-covalent interactions involving metal complexes may lead to the formation of aggregates (i.e., ground state dimers, trimers, n-mers, etc), and this is often linked to dramatic changes in their physical and chemical properties as compared to the original properties of the isolated units. Dimers and trimers can also be formed in the excited states potential energy surfaces, i.e., excimers. Excimers are short-lived but are also often characterized by different optical properties from those of the isolated units. Understanding the nature of non-covalent interactions and the presence or not of cooperativity effects in both aggregates and excimers is thus extremely important to rationalize these variations. In this study, we present computational investigations on isoquinolinyl pyrazolate Pt(II) complexes. Our results highlight that cooperativity effects between non-covalent interactions, which are modulated by steric-demanding substituents and metallophilic $\mathrm{Pt} \cdots \mathrm{Pt}$ interactions, are present only on certain investigated excimers. We use density functional theory (DFT) calculations to examine the cooperativity effects and the changes in the photophysical properties. Different descriptors of cooperativity effects between non-covalent interactions, including the synergetic, genuine non-additive, and total interaction energies were evaluated for a series of Pt(II) aggregates and excimers. In addition, energy decomposition analysis (EDA) calculations were performed to rationalize the origins of the cooperative effects. The cooperative effects in trimer excimers (in their lowest triplet excited state, i.e., $\mathrm{T}_{1}$ ) led to shortened $\mathrm{Pt} \cdots \mathrm{Pt}$ contacts as compared to the trimer aggregates. Furthermore, this synergy between non-covalent interactions is ultimately responsible for the formation of the excimers and the striking changes in the measured photophysical properties. More in detail, we report a change in the character of the lowest-lying triplet excited state when going from dimer excimers (i.e., of mixed triplet ligand-centered and triplet metal-to-ligand charge transfer $\left({ }^{3} \mathrm{LC} /{ }^{3} \mathrm{MLCT}\right)$ character) to trimer excimers (i.e., of triplet metal-metal-to-ligand charge transfer $\left({ }^{3} \mathrm{MMLCT}\right)$ character). The EDA reveals that the total interaction energy on trimer excimers is subtly controlled by the electrostatic and dispersion terms. 


\section{INTRODUCTION}

Non-covalent interactions are crucial in many areas of material science, biology, medicine, and modern chemistry, and especially in the field of supramolecular chemistry where they are responsible of the variations in the chemical and physical properties of single molecules when moving to condensed phases, for example, in liquid and solid states. ${ }^{1}$ Molecules are individual building blocks and the way in which they organize has a great impact on their chemical and physical properties. On the basis of the interacting molecules, cation- $\pi$ interactions, ${ }^{2-5}$ anion- $\pi$ interactions, ${ }^{6-9}$ lone pair interactions, ${ }^{10} \mathrm{CH} \cdots \pi$ interactions, ${ }^{11} \pi-\pi$ stacking interactions, ${ }^{11,12}$ and metallophilic interactions, ${ }^{13-17}$ have emerged as driving intermolecular forces in aggregated structures. In other scenarios, non-directional hydrophobic (i.e., dispersion-driven) interactions drive the formation of molecular aggregates. ${ }^{18}$ Particularly, the interactions involving aromatic rings are of key relevance in both chemical and biological recognition, i.e., they determine the structures of protein, DNA, and many other materials. In any process of aggregation, a large number of non-covalent interactions of different nature operate simultaneously, i.e., from electrostatic and induction to dispersion interactions. The interplay between non-covalent interactions may lead to synergistic or anti-synergistic effects. Cooperative (synergistic) effects between non-covalent interactions exist when the sum of the co-existing interactions in the supramolecular complex is larger than the simple addition of the individual interactions.

The cooperativity between $\pi-\pi$ interactions and other non-covalent interactions (i.e., ion- $\pi$, hydrogen bonding, etc) has been the scope of multiple investigations. ${ }^{19-31}$ The cooperative effects might be seen on the bonding distances between the molecular units, usually leading to shorter distances in the supramolecular complexes when synergistic effects arise. Scheme 1 displays e.g., the synergistic effects arising due to the co-existence of ion- $\pi$ and $\pi-\pi$ noncovalent interactions. In the latter case, a theoretical study highlighted that cooperative effects are mainly originated from induction effects, ${ }^{29}$ in which the ion- $\pi$ interaction induces a dipole moment at the benzene unit favoring its electrostatic interaction with another benzene unit. The cooperativity is reflected in the shortening of both the ion- $\pi$ and $\pi-\pi$ bond distances in the trimers, i.e., the $\pi-\pi$ distance is $3.7 \AA$ in the dimer, and it decreases to $3.5 \AA$ in the trimer. ${ }^{29}$ Accordingly, significant non-additivity energies were reported for these systems. ${ }^{20,28}$ Other scenarios involve the strengthening of hydrogen bonding interactions when co-existing with $\pi$ $\pi$ interactions and vice versa, ${ }^{20,32}$ but also the interplay between $\pi-\pi$ and $\mathrm{CH}-\pi$ interactions. ${ }^{33-}$ 38 


\section{Dimers}

\section{Trimer}

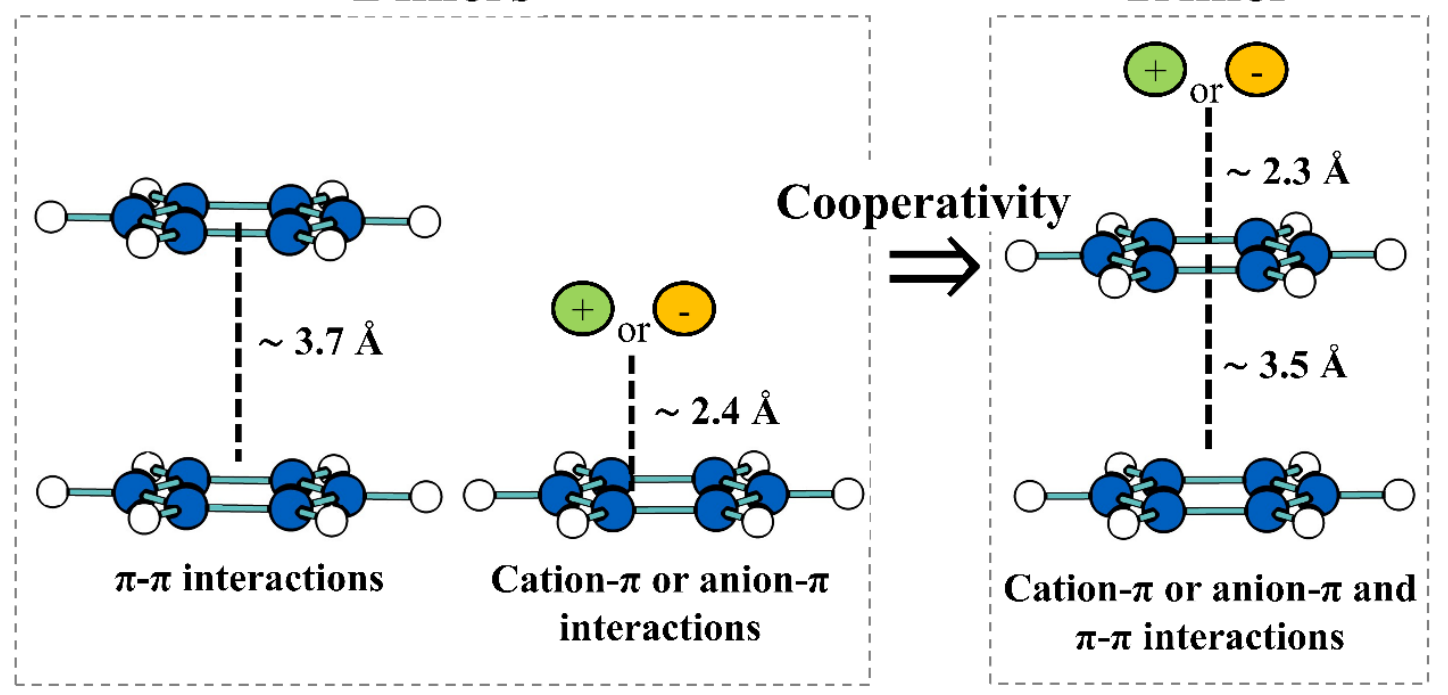

Cooperative energy $=\sim 0.6 \mathrm{kcal} / \mathrm{mol}$

Scheme 1. A representative cooperativity effect between non-covalent interactions reported in the literature. ${ }^{28}$ The main geometric parameters in $\AA$ (MP2/6-31G(d)) for the dimers and trimer along with the cooperative energy (MP2/6-311++G(d,p)//MP2/6-31G(d)) are highlighted.

For Pt(II) complexes, the interplay between non-covalent interactions resulting in cooperative effects were highlighted through several studies. ${ }^{39-43}$ For instance, Tam et. al. investigated the gelation properties of $\mathrm{Pt}(\mathrm{II})$ terpyridyl complexes. The gelation properties were found to be driven by a subtle interplay between Pt $\cdots \mathrm{Pt}$ and $\pi-\pi$ interactions in addition to hydrophobic interactions. ${ }^{39}$ Similarly, Rest et. al. reported an oligophenylene ethynylene-based amphiphilic Pt(II) complex that forms supramolecular polymeric structures in aqueous and polar media and which are driven by the interplay between $\pi-\pi$ and different weak $\mathrm{C}-\mathrm{H} \cdots \mathrm{Cl}$ or $\mathrm{C}-\mathrm{H} \cdots \mathrm{O}$ interactions. ${ }^{40}$ Furthermore, Suponitsky et. al. explored the effect of $\pi \cdots \pi$ stacking interactions on the hyperpolarizability of supramolecular materials. ${ }^{41}$ In most of the examples above, aggregates (i.e., ground state dimers and trimers) are formed. Dimers and trimers can also be formed in the excited state potential energy surfaces, those are known as excimers. Excimers, in contrast with aggregates, are short-lived, but can lead to striking changes in the measured photophysical properties. The formation of aggregates and excimers might be originated on subtle changes in the environment and thus their application in the field of chemosensors, bioprobes, stimuli-responsive nanomaterials, and optoelectronic materials is straightforward. ${ }^{17,39}$ The formation of excimer species is well described in $\mathrm{Pt}(\mathrm{II})$ complexes, but also in e.g., gold and silver complexes. ${ }^{44}$ Generally, these complexes display square planar 
molecular geometries originated from their $d^{8}$ electronic configuration, and thus favoring the formation of intermolecular metallophilic contacts along the sterically undemanding z-axis via $d-d$ orbital interactions. After light excitation of these complexes, relaxation on their excited state potential energy surfaces generally leads to the population of their lowest triplet excited state $\left(\mathrm{T}_{1}\right)$. Specifically for isoquinolinyl pyrazolate $\mathrm{Pt}(\mathrm{II})$ complexes, the initially populated singlet excited states of are mainly of mixed ligand-centered (LC) $\pi-\pi^{*}$ and metal-to-ligand charge transfer (MLCT) character. ${ }^{59}$ From these metal-based singlet excited states very fast and efficient intersystem crossing (ISC) mechanisms do take place leading to the population of the triplet manifold. Finally, relaxation on the triplet manifold leads to the population of the lowest-lying triplet excited states, which are responsible for the experimentally measured photoluminescent profiles with lifetimes expanding up to the microsecond regime. ${ }^{59}$ This manuscript focuses on these very last steps of the photorelaxation cascade.

In this regard, the formation of excimers is particularly notable for $\mathrm{Pt}(\mathrm{II})$ complexes bearing azolate chelates in solid states. ${ }^{45-48}$ The resulting triplet excimers often possess significant metal-metal-to-ligand charge transfer $\left({ }^{3} \mathrm{MMLCT}\right)$ character, in which the electron is excited from a filled $\mathrm{Pt} \cdots \mathrm{Pt} d_{z}^{2}$ orbital with certain antibonding $\sigma^{*}$ character to a vacant $\pi^{*}$ orbital of the chelate. The energy of the MMCLT excited states has a strong dependence on the Pt $\cdots \mathrm{Pt}$ interaction; i.e. shortened interlayer distances correlate with lower MMLCT energies. ${ }^{49}$ The metallophilic interactions can lead to the formation of dimers, trimers, oligomers or even onedimensional stacked structures, showing in some cases Pt $\cdots$ Pt separations shorter than the sum of van der Waals radii. ${ }^{13,15,50,51}$ Metallophilic Pt $\cdots$ Pt interactions are known to be comparable to the bond strength of hydrogen bonding, but are much weaker than that of typical covalent and ionic bonding. ${ }^{52}$ This means that these relatively weakened metallophilic interactions can be easily disturbed by both steric effect and other intra- or inter-molecular interactions, such as solvation, $\pi-\pi$ stacking, both hydrogen and halogen bonding interactions, etc; ${ }^{.53-57}$ but also by the global spin configuration of the molecular assembly $\left(\mathrm{S}_{0}, \mathrm{~T}_{1}\right)$. It also indicates that metallophilic interactions often coexist with other intermolecular forces that, in turn, would stabilize or destabilize the interlayer interactions.

For Pt(II) supramolecular structures and despite the growing understanding of their aggregates and excimers, most of the work has been directed towards tuning the $\pi \cdots \pi$ stacking and hydrogen bonding interactions. ${ }^{58}$ In a previous work, ${ }^{59}$ we reported square-planar Pt(II) complexes bearing isoquinolinyl pyrazolate with tunable photoluminescent properties (see Pt1-Pt4 in Scheme 2). The computed results revealed that first there are no short Pt••Pt 
contacts $(<3.0 \AA)$ on the ground state species and second that there is a certain degree of conformational flexibility on the formation of aggregates and excimers. A good correlation between the experimental and computed photophysical properties was reported in our previous publications; see e.g., a summary of the simulated UV-Vis absorption characteristics for Pt1 in Figure S5, where the red-shifted absorption bands due to singlet MMLCT excitations are only found for one specific trimer aggregate conformation. Concerning emission properties, the interlayer distance is the most important factor controlling the character of the excited states: for instance, only for Pt1 a trimer excimer of ${ }^{3}$ MMLCT character bearing short Pt $\bullet \bullet P t$ contacts is formed, while for the trimer excimers of Pt2-Pt4 their lowest triplet states are of mixed ${ }^{3} \mathrm{LC} /{ }^{3} \mathrm{MLCT}$ character. These results led us to explore whether cooperative effects between non-covalent interactions play a role in the formation of these excimers and to understand why dimer excimers of ${ }^{3}$ MMLCT character are apparently not formed. Specifically, using a first principles level approach, we first aim to understand the packing interactions in both aggregates and excimers of dimer and trimer species and to evaluate possible cooperativity effects when going from the dimer to the trimer species. The cooperative effects are analyzed in terms of geometric and energetic factors including the synergetic energy and the nonadditivity energy. Energy decomposition analysis (EDA) was used to decipher the origins of the synergistic effects between non-covalent interactions.

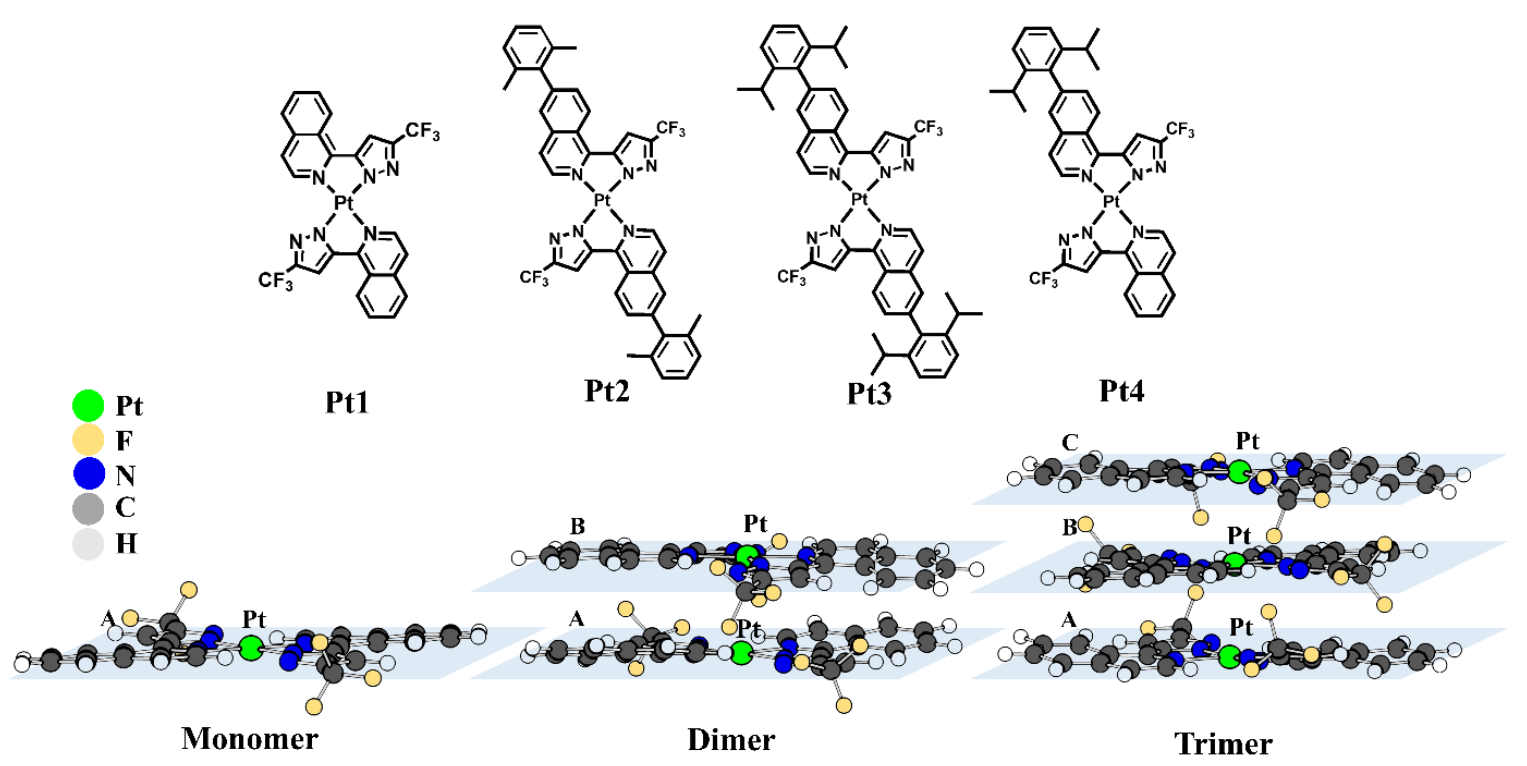

Scheme 2. Molecular geometries of monomers Pt1-Pt4 and schematic representation of monomer, dimer, and trimer structures. 


\section{COMPUTATIONAL DETAILS}

All calculations for Pt1-Pt4 are based on density functional theory (DFT). The geometries of Pt1-Pt4 in the singlet ground state $\left(\mathrm{S}_{0}\right)$ were optimized using the Becke's three-parameter hybrid exchange functional combined with the Lee-Yang-Parr correlation functional $^{60,61}$ including dispersion correction (B3LYP-D3), ${ }^{62}$ in combination with the 6-31G(d) atomic basis set for all non-metal atoms. Relativistic effects were considered for the metal Pt atoms by using the ECP-60-mwb pseudopotential. ${ }^{63}$ Geometries optimizations of the lowest triplet $\left(\mathrm{T}_{1}\right)$ states of Pt1-Pt4 were performed using unrestricted B3LYP-D3 (UB3LYP-D3) method using the same basis set as in the $\mathrm{S}_{0}$ optimizations. All optimized structures were confirmed to be local minima by normal mode analysis with all positive frequencies. All geometry optimizations were performed without symmetry constraints. The interaction energies of dimers and trimers are corrected for the basis set superposition error (BSSE) using the Boys-Bernardi counterpoise technique. ${ }^{64}$ The geometry optimizations of Pt1-Pt4 were carried out with the Gaussian 16 program package. ${ }^{65}$

To evaluate the cooperativity between non-covalent interactions, the synergetic energies $\left(E_{\text {syn }}\right)$ of Pt1-Pt4 were performed by subtracting the binding energy of dimers $\left(E_{\text {dimer }}\right)$ from the binding energy of the trimers $\left(E_{\text {trimer }}\right)$ as displayed in Equation (1). Their genuine non-additivity energies $\left(E-E_{\mathrm{A}}\right)$ were also calculated by deducting all pairwise interaction (three in the case of a ternary complex) from the total interaction energy of trimers as given in Equation (2).

$$
\begin{aligned}
& E_{\text {syn }}=E_{\text {trimer }}-2\left(E_{\text {dimer }}\right) \\
& E-E_{A}=E_{A B C}-\left(E_{A B}+E_{A C}+E_{B C}\right)
\end{aligned}
$$

Where $E_{\mathrm{ABD}}, E_{\mathrm{AB}}, E_{\mathrm{AC}}, E_{\mathrm{BC}}$ terms correspond to the interaction energies of $\mathrm{ABC}, \mathrm{AB}, \mathrm{AC}$, and $\mathrm{BC}$, respectively, at the optimized $\mathrm{ABC}$ trimer geometries. The $\mathrm{ABC}$ species is composed of three layers of the monomer unit, i.e., $\mathrm{A}, \mathrm{B}$, and $\mathrm{C}$ as displayed in Scheme 2. The $\mathrm{AB}, \mathrm{AC}$, and $\mathrm{BC}$ species correspond to the respective dimer combinations at the optimized $\mathrm{ABC}$ trimer geometries. These expressions have been successfully used in the study of cooperativity effects among a variety of systems such as the interplay between ion- $\pi$ and $\pi-\pi$ interactions or between $\mathrm{C}-\mathrm{H} / \pi$ and $\pi-\pi$ interactions. ${ }^{20,28,31}$

We have also performed energy decomposition analysis (EDA) to evaluate the contribution of various factors to the total interaction energies. ${ }^{66}$ The EDA of dimers and trimers Pt1-Pt4 were performed based on the generalized Kohn-Sham (GKS) scheme, i.e., GKS-EDA, as implemented in the Turbomole package. ${ }^{67,68}$ This scheme divides the total interaction energy 
$\left(\Delta E_{\mathrm{TOT}}\right)$ into electrostatic $\left(\Delta E_{\text {ele }}\right)$, exchange-repulsion $\left(\Delta E_{\text {exrep }}\right)$, polarization $\left(\Delta E_{\mathrm{pol}}\right)$, correlation $\left(\Delta E_{\text {corr }}\right)$, and dispersion $\left(\Delta E_{\text {disp }}\right)$ terms as follows in Equation (3).

$$
\Delta E_{T O T}=\Delta E_{\text {ele }}+\Delta E_{\text {exrep }}+\Delta E_{\text {pol }}+\Delta E_{\text {corr }}+\Delta E_{\text {disp }}
$$

The individual terms are defined in the SI. The EDA calculations were performed with the Turbomole 7.1 program package ${ }^{69}$ using the same version of the B3LYP-D3 functional as implemented in Gaussian 16 and the def2-TZVP basis set. ${ }^{70}$

\section{RESULTS AND DISCUSSION}

Optimized Structures. The optimized geometries in the $\mathrm{S}_{0}$ state of monomers Pt1-Pt4 are shown in Figure 1 (see Figure $\mathrm{S} 1$ in the Supporting Information for the $\mathrm{T}_{1}$ optimized geometries). The $\mathrm{S}_{0}$ and $\mathrm{T}_{1}$ optimized geometries of dimers and trimers of Pt1 are displayed in Figure 2 (see Figure S2-S4 and Table S1-S2 in the Supporting Information for the rest of complexes). We note that through the analysis of the optimized geometries in the multimers one can already assess whether the formation aggregates $\left(\mathrm{S}_{0}\right)$ and of excimers $\left(\mathrm{T}_{1}\right)$ is likely.

Let us start with the monomers. All monomer Pt(II) complexes adopt a square-planar coordination of the central Pt(II) atom, with the ligands adopting a trans-configuration. The $\mathrm{Pt}-\mathrm{N}$ bond lengths are almost the same without obvious variation for all monomers.

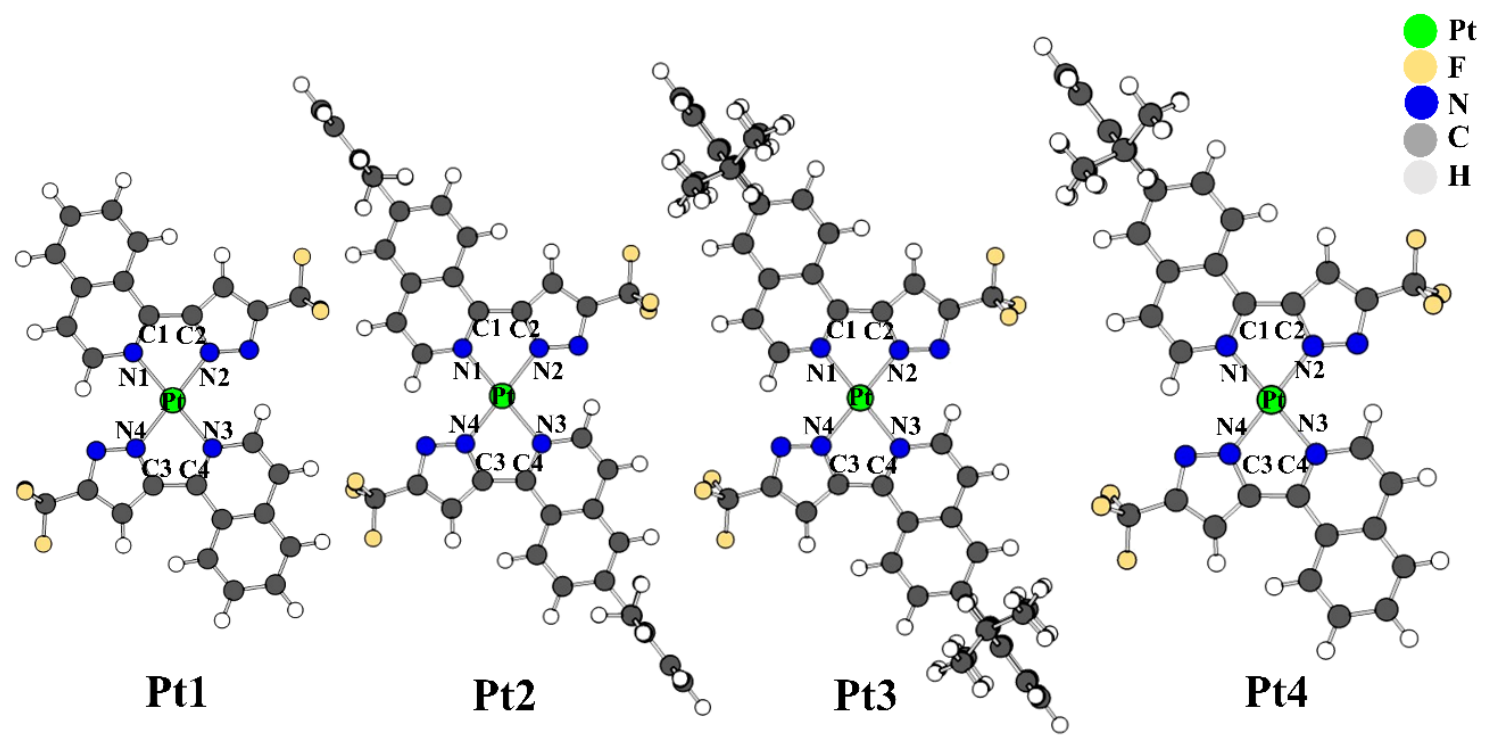

Figure 1. Optimized geometries (B3LYP-D3/6-31G(d)) in the $\mathrm{S}_{0}$ state of monomers of Pt1-Pt4.

In the case of dimers and trimers, the geometries of different conformers in the $\mathrm{S}_{0}$ and $\mathrm{T}_{1}$ potential energy surfaces were optimized. Figure 2 and Figure S2-S4 in the Supporting 
Information collect: i) the geometries of all the optimized conformers (see e.g., Pt1a-c in Figure 2), ii) their Pt $\cdots P t$ distances, and iii) their relative energies; both for dimers and trimers. Several conformers are found to be close-lying both in the $\mathrm{S}_{0}$ and $\mathrm{T}_{1}$ potential energy surfaces (e.g., within $0.0-2.0 \mathrm{kcal} / \mathrm{mol}$ ), and thus, likely to be populated at room temperature if thermal equilibrium is at play. These results highlight that there is some degree of conformational flexibility for dimers and trimers at room temperature. Two main intermolecular forces drive the dimers and trimers assembly: intermolecular $\pi-\pi$ stacking interactions and Pt $\cdots \mathrm{Pt}$ contacts. The former interaction is predominant in all investigated dimer species, regardless whether we consider the $\mathrm{S}_{0}$ or the $\mathrm{T}_{1}$ optimized geometries. The interlayer distances range between 3.3 and $3.6 \AA$ (see Figure 2). For example, the computed interlayer distance for the lowest-energy $\mathrm{S}_{0}$ dimer of Pt1 is $3.59 \AA$ (see Pt1b). Short Pt $\cdots P t$ contacts $(<3.0 \AA)$ are only found in the case of selected $\mathrm{T}_{1}$ optimized conformers; thus, highlighting that the latter interactions might be feasible only in the excimers but not in the aggregates. Furthermore, this is only observed in the trimer excimers but not on the dimer excimers. For example, let us evaluate the case of the excimers of Pt1. The lowest-energy $\mathrm{T}_{1}$ conformer of the dimer of Pt1 displays an interlayer distance of $3.56 \AA$ (see Pt1c). This distance turns significantly shorter in the lowest-energy $\mathrm{T}_{1}$ conformer of the trimer (see $2.94 \AA$ for Pt1a), where the Pt $\cdots$ Pt contacts become more evident. The other $\mathrm{T}_{1}$ trimer conformers possess significant larger interlayer distances (3.53 and $3.52 \AA$ for Pt1b and Pt1c respectively) and they are located ca. 5.4 and $4.9 \mathrm{kcal} / \mathrm{mol}$, respectively, above Pt1a. Thus, we foresaw that cooperativity between metallophilic interactions might be relevant to rationalize the relevant geometric and energetic differences between the conformers. We recall that these changes are associated to the striking change in their photophysical properties from very diluted solutions to concentrated dilutions or thin films, i.e., yielding red shifted-emission bands and larger photoluminescence quantum yields in the films. ${ }^{59}$ 


\section{(a) Dimer}
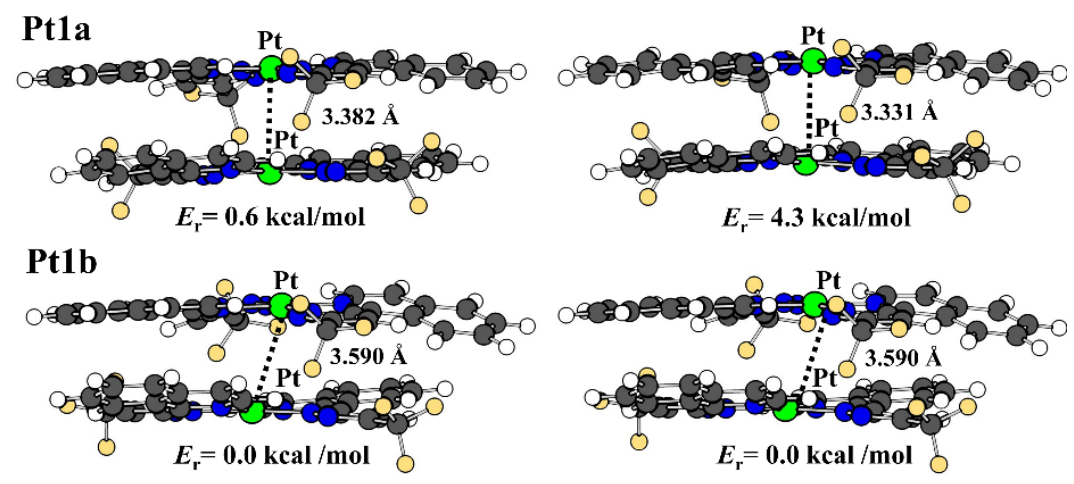

Pt1c

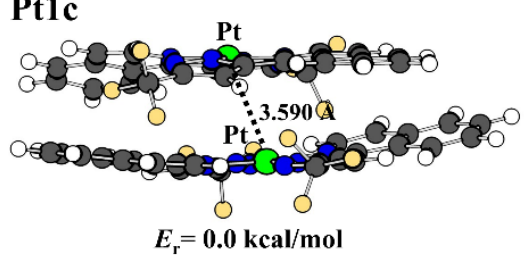

$S_{0}$ state
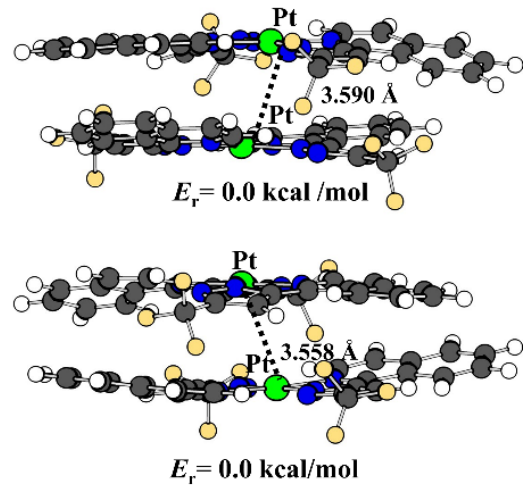

$T_{1}$ state

(b) Trimer
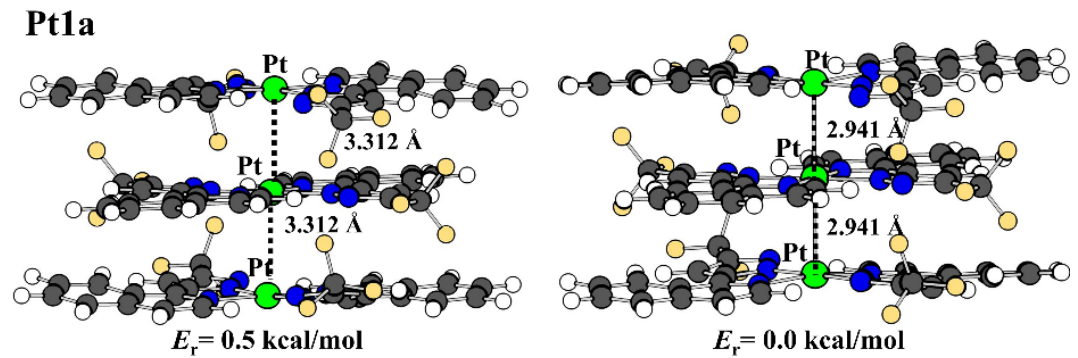

Pt1b

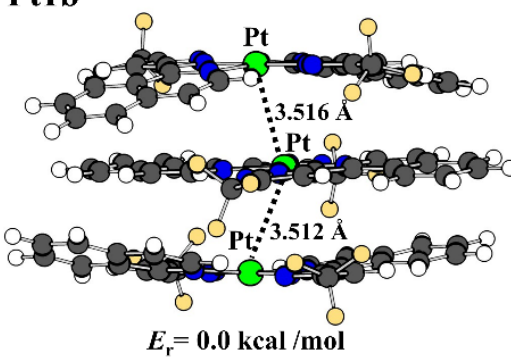

Pt1c

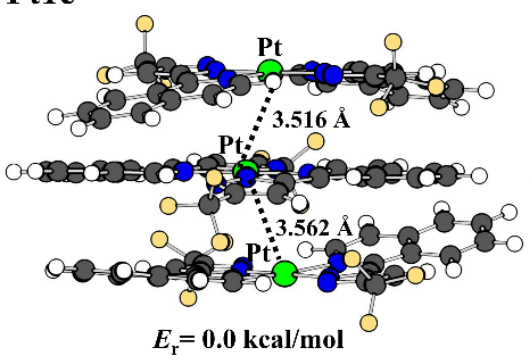

$\mathrm{S}_{0}$ state
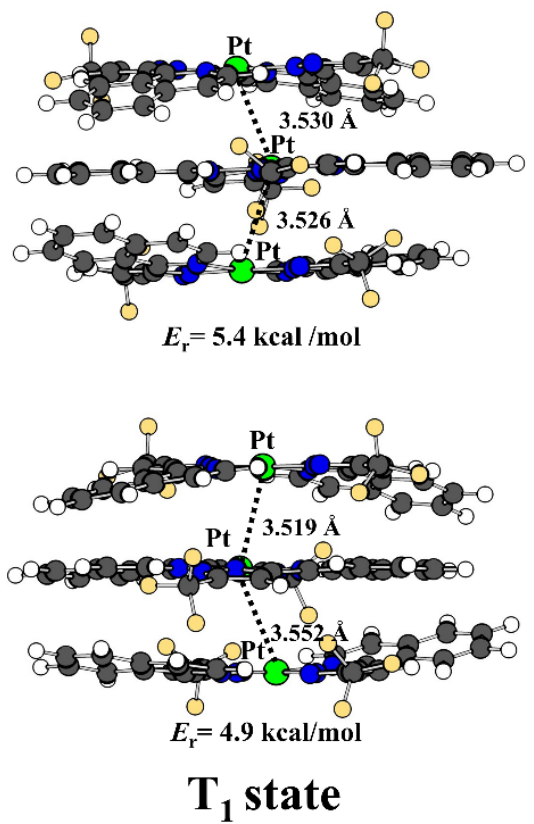

Figure 2. Optimized conformers (B3LYP-D3/6-31G(d)) in the $\mathrm{S}_{0}$ and $\mathrm{T}_{1}$ states of dimers and trimers of Pt1 along with their relative energies $\left(E_{\mathrm{r}}\right)$. 
Estimation of Cooperativity between Non-Covalent Interactions. In Table 1 and Table 2 , we summarize the binding energies with and without the basis set superposition error (BSSE) correction ( $E_{\mathrm{BSSE}}$ and $E$, respectively) and the equilibrium distance $\left(R_{\mathrm{e}}\right.$ in $\AA$ ) of dimers Pt1-Pt4 in their $\mathrm{S}_{0}$ and $\mathrm{T}_{1}$ states; and trimers of Pt1 in their $\mathrm{S}_{0}$ and $\mathrm{T}_{1}$ states (see Table S3-S4 in the Supporting Information for the rest of complexes), respectively. The results show that, not surprisingly, the trimer species are more stable than dimer species. For example, the binding energy of Pt1a in the $\mathrm{S}_{0}$ state is increased from $-39.0 \mathrm{kcal} / \mathrm{mol}$ in the dimer structure to -76.5 $\mathrm{kcal} / \mathrm{mol}$ in the trimer structure. For dimers, the binding energies of the aggregates $\left(\mathrm{S}_{0}\right)$ are on the same order of magnitude as those in the excimers $\left(T_{1}\right)$, see Table 1. However, in the case of trimers, the formation of excimers is clearly more favored than in the case of aggregates (compare in Table $2 E_{\mathrm{BSSE}}$ values ranging between ca. $-80 /-95 \mathrm{kcal} / \mathrm{mol}$ for the $\mathrm{T}_{1}$ species with respect to ca. $-76 /-79 \mathrm{kcal} / \mathrm{mol}$ for the $\mathrm{S}_{0}$ species).

To provide an estimation of the extra energetic stabilization or destabilization in trimer species with respect to the dimers, we present the computed values of synergetic $\left(E_{\text {syn }}\right)$ and genuine non-additivity $\left(E-E_{\mathrm{A}}\right)$ energies for Pt1 in Table 2 (see Table S4 in the Supporting Information for trimers Pt2-Pt4). These energies are intended to provide an estimation of energetic stabilization or destabilization obtained in trimer species. The $E_{\text {syn }}$ is the simplest quantity, which is obtained by subtracting twice the $E_{\text {dimer }}$ to the $E_{\text {trimer }}$. Another possibility for evaluating the mutual influence of both interactions in trimer species is $E$ - $E_{\mathrm{A}}$, which is an energy difference between the $E$ of trimer species and all pair of interaction energies in the supramolecular trimers (see SI).

The computed values of $E_{\mathrm{syn}}$ and $E-E_{\mathrm{A}}$ of trimers Pt1 are collected in Table 2 (see Table S4 in the Supporting Information for the rest of complexes). An inspection of the results reveals that the $E_{\mathrm{syn}}$ and $E-E_{\mathrm{A}}$ of trimer species are close to zero or slightly negligible in the ground state (values ranging between -3.0 and $0.0 \mathrm{kcal} / \mathrm{mol}$ for all possible aggregates), and thus highlighting that cooperative effects are likely not playing a role in the ground state structures. We recall that the computed interlayer distances do not strongly vary when going from dimer to trimer (compare 3.382 vs $3.312 \AA$ in Pt1a, see Figure 2-3). However, for the excimers the interlayer distance is strongly shortened when going from dimers to trimers for some of the optimized triplet conformers (see e.g., $2.941 \AA$ in Pt1a in Figure 2). Interestingly, this latter conformer displaying unambiguous $\mathrm{Pt} \cdots \mathrm{Pt}$ contacts, becomes the lowest-lying conformer among all the optimized triplet state structures. Thus, the computed geometric features at the trimer excimers point to strong cooperativity effects when going from dimers to trimers. Indeed, the computed $E_{\mathrm{syn}}$ and $E-E_{\mathrm{A}}$ values also highlight the strong cooperative effects in Pt1a 
(see Table 2). Specifically, while the $E_{\mathrm{syn}}$ and $E-E_{\mathrm{A}}$ values for trimer Pt1a in $\mathrm{S}_{0}$ are negligible; they amount up to -16.9 and $-8.2 \mathrm{kcal} / \mathrm{mol}$ in the $\mathrm{T}_{1}$ state, respectively. Moreover, for the higher-lying energy conformers in the $\mathrm{T}_{1}$ state, i.e., Pt1b-c, smaller cooperative effects are calculated ( $E_{\text {syn }}$ values of -15.0 and $-1.8 \mathrm{kcal} / \mathrm{mol}$ ). This is in agreement with the fact that the computed interlayer distances do not strongly vary when going from dimers to trimers for these conformers. In a nutshell, these pieces of evidence indicate a positive synergy between noncovalent interactions for excimers, and especially for the lowest energy conformer of the $T_{1}$ state. This trend is not only observed for Pt1a but can also be seen in the rest of complexes (see Table S4 in the Supporting Information).

Table 1. Binding energies (B3LYP-D3/def2-TZVP//B3LYP-D3/6-31G(d)) with and without BSSE correction $\left(E_{\mathrm{BSSE}}\right.$ and $E$ in $\left.\mathrm{kcal} / \mathrm{mol}\right)$ and $\mathrm{Pt} \cdots \mathrm{Pt}$ equilibrium distance $\left(R_{\mathrm{e}}\right.$ in $\AA$ ) of the dimer conformers of Pt1 in their $\mathrm{S}_{0}$ and $\mathrm{T}_{1}$ states.

\begin{tabular}{cccccccc}
\hline \multirow{2}{*}{ Dimer } & \multicolumn{3}{c}{$\mathrm{S}_{0}$ state } & & \multicolumn{3}{c}{$\mathrm{T}_{1}$ state } \\
\cline { 2 - 4 } \cline { 6 - 7 } & $E$ & $E_{\mathrm{BSSE}}$ & $R_{\mathrm{e}}$ & & $E$ & $E_{\mathrm{BSSE}}$ & $R_{\mathrm{e}}$ \\
\hline Pt1a & -41.7 & -38.9 & 3.382 & & -40.5 & -39.0 & 3.331 \\
Pt1b & -42.8 & -39.7 & 3.590 & & -42.9 & -39.3 & 3.558 \\
Pt1c & -43.0 & -40.2 & 3.590 & & -42.9 & -39.3 & 3.552 \\
\hline
\end{tabular}

Table 2. Binding energies (B3LYP-D3/def2-TZVP//B3LYP-D3/6-31G(d)) with and without BSSE correction $\left(E_{\mathrm{BSSE}}\right.$ and $\left.E\right)$, synergetic $\left(E_{\mathrm{syn}}\right)$, genuine non-additivity $\left(E-E_{\mathrm{A}}\right)$ energies in $\mathrm{kcal} / \mathrm{mol}$ and $\mathrm{Pt} \cdots \mathrm{Pt}$ equilibrium distances $\left(R_{\mathrm{e} 1}\right.$ and $R_{\mathrm{e} 2}$ in $\AA$ ) of the trimer conformers of Pt1 in their $\mathrm{S}_{0}$ and $\mathrm{T}_{1}$ states.

\begin{tabular}{|c|c|c|c|c|c|c|c|c|c|c|c|c|}
\hline \multirow{2}{*}{ Trimer } & \multicolumn{6}{|c|}{$\mathrm{S}_{0}$ state } & \multicolumn{6}{|c|}{$\mathrm{T}_{1}$ state } \\
\hline & $E$ & $E_{\mathrm{BSSE}}$ & $E_{\mathrm{syn}}$ & $E-E_{\mathrm{A}}$ & $R_{\mathrm{e} 1}$ & $R_{\mathrm{e} 2}$ & $E$ & $E_{\mathrm{BSSE}}$ & $E_{\text {syn }}$ & $E-E_{\mathrm{A}}$ & $R_{\mathrm{e} 1}$ & $R_{\mathrm{e} 2}$ \\
\hline Pt1a & -81.9 & -76.5 & 1.3 & -2.1 & 3.312 & 3.312 & -100.0 & -94.9 & -16.9 & -8.2 & 2.941 & 2.941 \\
\hline Pt1b & -84.8 & -79.4 & 0.0 & -2.9 & 3.516 & 3.512 & -96.9 & -93.6 & -15.0 & -3.1 & 3.530 & 3.526 \\
\hline Pt1c & -84.8 & -79.5 & 0.9 & -2.2 & 3.516 & 3.562 & -85.7 & -80.4 & -1.8 & -2.1 & 3.519 & 3.552 \\
\hline
\end{tabular}

In Figure 3a are depicted the spin density plots for the dimer and trimer excimers of Pt1a. Triplet excited state species dominate the emissive properties of these complexes, as we recently disclosed both experimentally and computationally. ${ }^{59}$ When moving from dimer to trimer excimer the character of $\mathrm{T}_{1}$ state changes from mixed ${ }^{3} \mathrm{LC} /{ }^{3} \mathrm{MLCT}$ character to ${ }^{3}$ MMLCT. As discussed in our previous report, ${ }^{59}$ this change of character is accompanied by striking changes in the photophysical properties when moving from very diluted solutions to 
concentrated dilutions or thin films (i.e., red-shifted emission peaks, higher photoluminescence quantum yields, etc.). Specifically, in our previous report (see a summary of the computed emission energies in Table S5 for all the complexes), ${ }^{59}$ for trimer Pt1a (which possesses ${ }^{3} \mathrm{LC} /{ }^{3} \mathrm{MMLCT}$ character) an emission band peaking at $842 \mathrm{~nm}$ was reported. This band is redshifted in comparison with the computed energy maxima for trimers Pt1b and Pt1c which possess mixed ${ }^{3} \mathrm{LC} /{ }^{3} \mathrm{MLCT}$ character (i.e., 755 and $788 \mathrm{~nm}$; respectively) but also with respect of dimers Pt1b and Pt1c (749 nm). In opposition to trimer-emission, dimer-emission resembles more to the monomer emission since their responsible emissive states are also of ${ }^{3} \mathrm{LC} /{ }^{3} \mathrm{MLCT}$ character. In Figure $3 b$ are displayed the spin density plots for the the trimer conformers of Pt1. In contrast to the trimer of Pt1a, both $\mathrm{T}_{1}$ states of trimers Pt1b and Pt1c can be better described as having mixed ${ }^{3} \mathrm{LC} /{ }^{3} \mathrm{MLCT}$ character. Thus, having in mind that these conformers are energetically close-lying, we can rationalize why e.g., subtle changes in the environment may lead to the formation of aggregates in the excited state potential energy surfaces, as observed experimentally. ${ }^{59}$

(a)

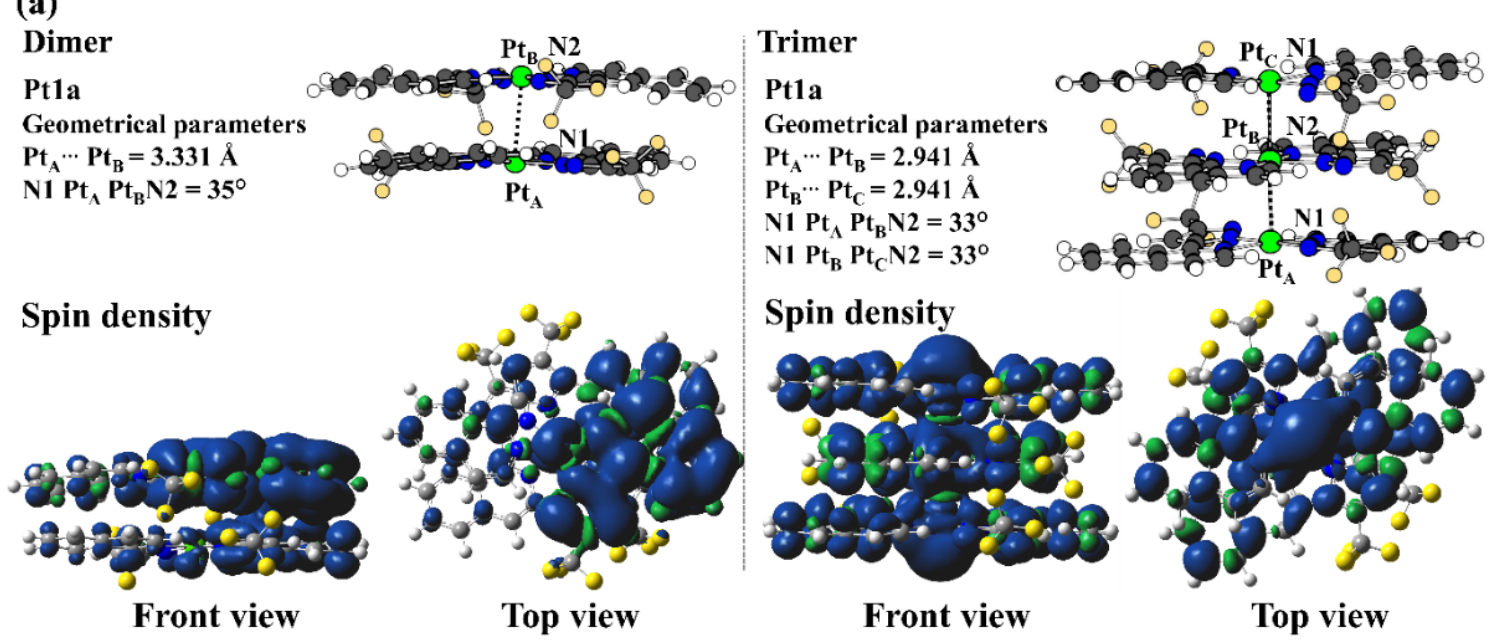

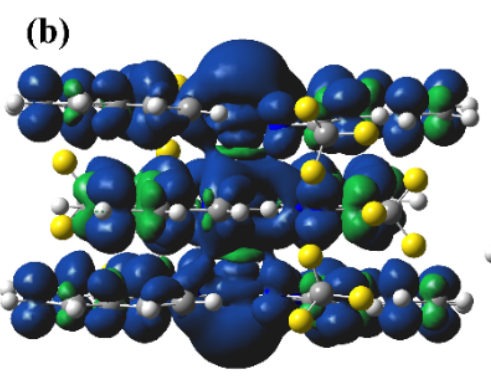

Pt1a

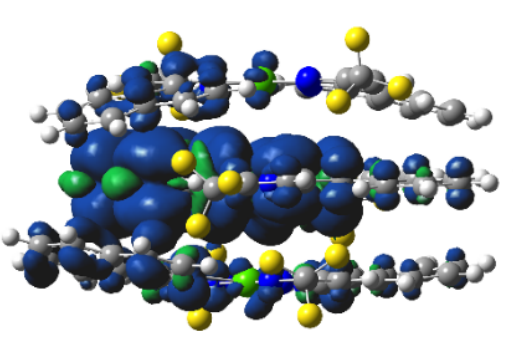

Pt1b

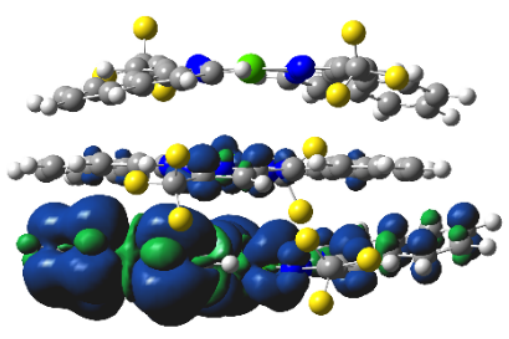

Pt1c 
Figure 3. (a) Optimized geometries (B3LYP-D3/6-31G(d)) and spin density distributions of dimer and trimer excimers of Pt1a and (b) spin density distributions of trimers Pt1a-c (Isocontour value 0.0002 : blue for alpha spin and green for beta spin). 
Energy Decomposition Analysis. To provide an estimation of the extra energetic stabilization obtained in the excimers of the supramolecular complexes when going from the dimer to the trimer species, and to obtain a rationalization of the origins of this cooperativity, we performed energy decomposition analysis (EDA) calculations as given in Equation (3). The analysis was performed for all dimer conformers of Pt1-Pt4 and for the trimer conformers of Pt1, in both their $\mathrm{S}_{0}$ and $\mathrm{T}_{1}$ states. Figure 4 depicts the possible fragmentation schemes that we have used. While the fragmentation of dimers is straightforward (see Figure 4a), several fragmentation schemes are possible for the trimers, especially for the $T_{1}$ state. Specifically, for the excimer trimers, layers A, B and C can be spin restricted or spin unrestricted (the total wavefunction is maintained as spin unrestricted). Furthermore, layers $\mathrm{A}$ and $\mathrm{C}$ also can be treated as the first fragment and the middle (B) layer can be treated as a second fragment where they can be spin restricted or spin unrestricted. This leads to five different possibilities for excimer trimers (Type I-V, see Figure 4b-c), which were all analysed in the EDA calculations for comparative purposes.

(a)
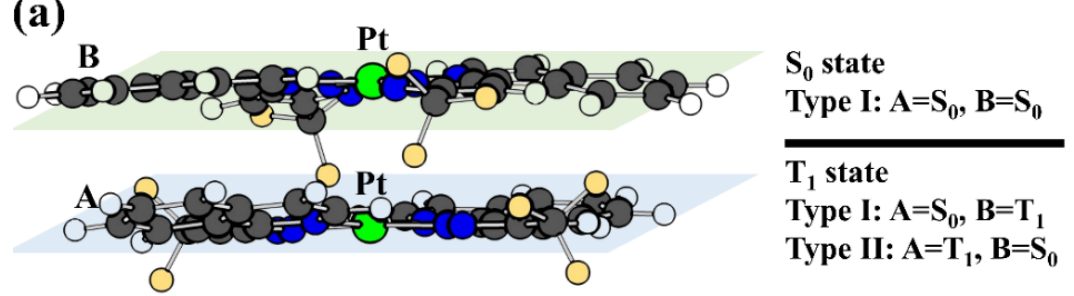

(b)

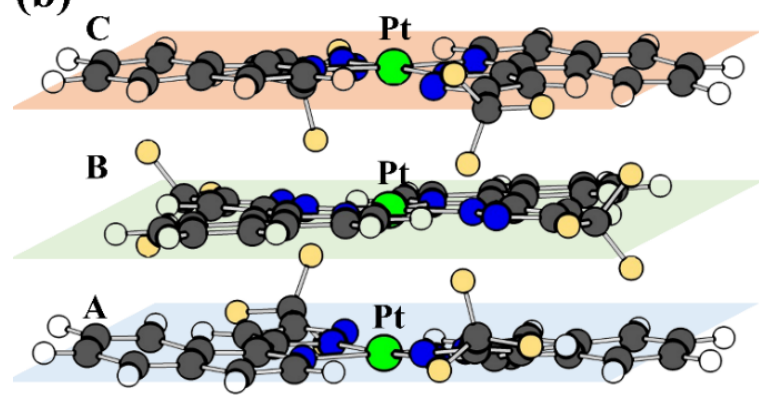

$S_{0}$ state

Type I: $\mathrm{A}=\mathrm{S}_{0}, \mathrm{~B}=\mathrm{S}_{0}, \mathrm{C}=\mathrm{S}_{\mathbf{0}}$

$T_{1}$ state

Type I: $\mathrm{A}=\mathrm{S}_{0}, \mathrm{~B}=\mathrm{S}_{\mathbf{0}}, \mathrm{C}=\mathrm{T}_{1}$ Type II: $A=S_{0}, B=T_{1}, C=S_{0}$

(c)

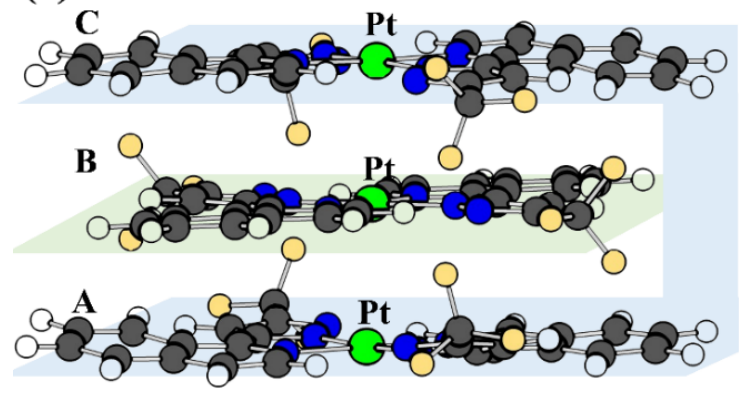

$\mathrm{S}_{0}$ state

Type II: $A=S_{0}, B=S_{0}, C=S_{0}$

$\mathrm{T}_{1}$ state

Type IV: $\mathrm{AC}=\mathrm{S}_{0}, \mathrm{~B}=\mathrm{T}_{1}$

Type V: $A C=T_{1}, B=S_{0}$ 
Figure 4. Fragmentation schemes used in EDA calculations; (a) dimers Pt1, (b) trimers Pt1 using a three-layer fragmentation, and (c) trimers Pt1 in which the terminal monomers (AC) is treated as the first fragment and the middle monomer (B) as second fragment.

Firstly, GKS-EDA calculations were done on all optimized conformers in both their $\mathrm{S}_{0}$ and $\mathrm{T}_{1}$ states. The results for the dimers and trimers of $\mathbf{P t 1}$ are listed in Table 3 (see Table S6 in the Supporting Information for the rest of complexes). Secondly, to evaluate how the global EDA total interaction energy $\left(\Delta E_{\text {tot }}\right)$ and its different energetic contributions vary along the Pt $\cdots$ Pt bond distances, potential energy curves were computed for both dimers and trimers of Pt1 as shown in Figure 5. For the latter studies, the conformer Pt1a was selected to evaluate the changes when going from dimer to trimer. In the case of trimers, the two Pt $\cdots$ Pt bond distances were symmetrically distorted to build up the potential energy curves.

Let us start the discussion with the values reported in Table 3. First, the dispersion contribution $\left(\Delta E_{\text {disp }}\right)$ dominates the EDA total interaction energies $\left(\Delta E_{\text {tot }}\right)$ for both dimers and trimers in their $\mathrm{S}_{0}$ or $\mathrm{T}_{1}$ electronic configurations. The next terms contributing to binding are the $\Delta E_{\text {exrep }}$ and $\Delta E_{\text {corr }}$ terms. The $\Delta E_{\text {ele }}$ contribution is always repulsive (see positive values in Table 3), which can be understood in terms of the electrostatic repulsion between the Pt(II) units, while the polarization term $\left(\Delta E_{\mathrm{pol}}\right)$ remains negligible in all the situations. Therefore, a first inspection of the results clearly reveals that the binding situation is controlled by the electrostatic and dispersion terms, and modulated by the $\Delta E_{\text {exrep }}$ and $\Delta E_{\text {corr }}$ terms. Let us now discuss the results in more details. For instance, for the dimer excimer $\left(\mathrm{T}_{1}\right)$ of Pt1a, the BSSEcorrected dimer binding energy $\left(E_{\text {tot(BSSE) }}\right.$ in Table 1$)$ is ca. $-39.0 \mathrm{kcal} / \mathrm{mol}$. Values of ca. -39 $\mathrm{kcal} / \mathrm{mol}$ are obtained for the BSSE-corrected GKS-EDA $\Delta E_{\text {tot }}$ values ( $\Delta E_{\text {tot(BSSE) }}$ in Table 3$)$, both for type I and II fragmentations, so that the results are consistent regardless of the considered fragmentation scheme and we thus use here type I fragmentation for Pt1b and Pt1c. Note that the $\Delta E_{\text {tot(BSSE) }}$ values for the latter compounds amount up to $-39.3 \mathrm{kcal} / \mathrm{mol}$ in their $\mathrm{T}_{1}$ states, values also in agreement with those reported in Table 1.

In the case of the trimer Pt1a, the $\Delta E_{\text {tot(BSSE) }}$ values slightly vary between the different fragmentation schemes. For instance, type III and IV provide similar $\Delta E_{\text {tot(BSSE) values of }}$ -94.9 and $-94.2 \mathrm{kcal} / \mathrm{mol}$, respectively, whereas type I, II, and V render values of $\mathrm{f}-94.1,-91.4$, and $-96.4 \mathrm{kcal} / \mathrm{mol}$, respectively. Because the computed binding energy is $-94.9 \mathrm{kcal} / \mathrm{mol}$ (see Table 2), the analysis here is based on type III and IV fragmentations. For trimers Pt1b and Pt1c the type III fragmentation leads also to the most similar results with respect to the values 
reported in Table 2, and thus this type of fragmentation is chosen for comparative purposes. By comparing the trimers Pt1a, Pt1b and Pt1c in their $T_{1}$ states, one can notice that the shortening of the Pt $\cdots \mathrm{Pt}$ distance in Pt1a has a strong influence on the $\Delta E_{\text {ele }}$ term, which increases from ca. 169.7 and $170.4 \mathrm{kcal} / \mathrm{mol}$ in Pt1b and Pt1c, respectively, to ca. 179.3 $\mathrm{kcal} / \mathrm{mol}$ in Pt1a. This is as expected due to the increasing electrostatic repulsion with decreasing interlayer distances. Conversely, a more favorable dispersion term $\left(\Delta E_{\text {disp }}\right)$ is obtained for the latter (compare ca. $-131.9 \mathrm{kcal} / \mathrm{mol}$ for Pt1a versus -125.5 and $-125.1 \mathrm{kcal} / \mathrm{mol}$ for Pt1b and Pt1c, respectively). Therefore, the subtle variations in the $\Delta E_{\text {exrep }}$ and $\Delta E_{\text {corr }}$ terms determine the total binding energies, since the $\Delta E_{\mathrm{pol}}$ remains in all cases negligible. Particularly, the striking changes in the $\Delta E_{\text {corr }}$ term within the series of conformers are very relevant to explain the observed trends in the $\Delta E_{\text {tot }}$ values. Thus, the $\Delta E_{\text {corr }}$ term is more beneficial in the case of Pt1a (-72.6 kcal/mol) than in the case of Pt1b and Pt1c (-62.6 and $62.2 \mathrm{kcal} / \mathrm{mol}$, respectively). Due to the shortened interlayer distances correlation effects are also more relevant in Pt1a, which finally leads to a larger total energy for Pt1a as compared to those of Pt1b and Pt1c. Concerning the $\Delta E_{\text {exrep }}$ values, they amount up to ca. -75.1, -79.1 and -69.3 kcal/mol for Pt1b, Pt1a and Pt1c, respectively. In a nutshell, while dispersion contributions are mainly responsible for the binding energies, the subtle changes in the $\Delta E_{\text {disp, }}$ $\Delta E_{\text {exrep }}, \Delta E_{\text {corr }}$ and $\Delta E_{\text {ele }}$ terms need to be concomitantly analyzed in order to understand the trends in the binding energies.

Table 3. Total interaction energies with and without BSSE correction $\left(\Delta E_{(\mathrm{BSSE})}\right.$ and $\left.\Delta E\right)$, electrostatic $\left(\Delta E_{\text {ele }}\right)$, exchange-repulsion $\left(\Delta E_{\text {exrep }}\right)$, polarization $\left(\Delta E_{\mathrm{pol}}\right)$, correlation $\left(\Delta E_{\text {corr }}\right)$, and dispersion $\left(\Delta E_{\text {disp }}\right.$ ) energies in $\mathrm{kcal} / \mathrm{mol}$ of dimer and trimer conformers of Pt1 in their $\mathrm{S}_{0}$ and $\mathrm{T}_{1}$ states computed at B3LYP-D3/def2-TZVP//B3LYP-D3/6-31G(d) level.

\begin{tabular}{|c|c|c|c|c|c|c|c|c|c|}
\hline \multicolumn{3}{|c|}{ Pt1 } & $\Delta E_{\text {ele }}$ & $\Delta E_{\text {exrep }}$ & $\Delta E_{\mathrm{pol}}$ & $\Delta E_{\text {corr }}$ & $\Delta E_{\text {disp }}$ & $\Delta E_{\text {tot }}$ & $\Delta E_{\mathrm{tot}(\mathrm{BSSE})}$ \\
\hline \multirow[t]{9}{*}{$\overline{\text { Dimer }}$} & \multicolumn{8}{|c|}{$\mathrm{S}_{0}$ state } & \\
\hline & Pt1a & & 85.1 & -33.9 & 0.0 & -31.8 & -60.6 & -41.2 & -38.9 \\
\hline & Pt1b & & 83.5 & -34.2 & 0.0 & -31.3 & -61.0 & -43.0 & -39.7 \\
\hline & Pt1c & & 83.4 & -34.2 & 0.0 & -31.3 & -61.1 & -43.2 & -40.2 \\
\hline & \multicolumn{8}{|c|}{$\mathrm{T}_{1}$ state } & \\
\hline & \multirow[t]{2}{*}{ Pt1a } & $\bar{I}$ & 89.8 & -33.8 & 0.0 & -35.0 & -63.6 & -42.6 & -39.9 \\
\hline & & II & 90.7 & -34.9 & 0.0 & -35.0 & -61.5 & -40.7 & -39.0 \\
\hline & Pt1b & $\bar{I}$ & 83.0 & -32.7 & 0.0 & -31.9 & -61.5 & -43.1 & -39.3 \\
\hline & Pt1c & I & 83.0 & -32.7 & 0.0 & -31.9 & -61.5 & -43.1 & -39.3 \\
\hline
\end{tabular}




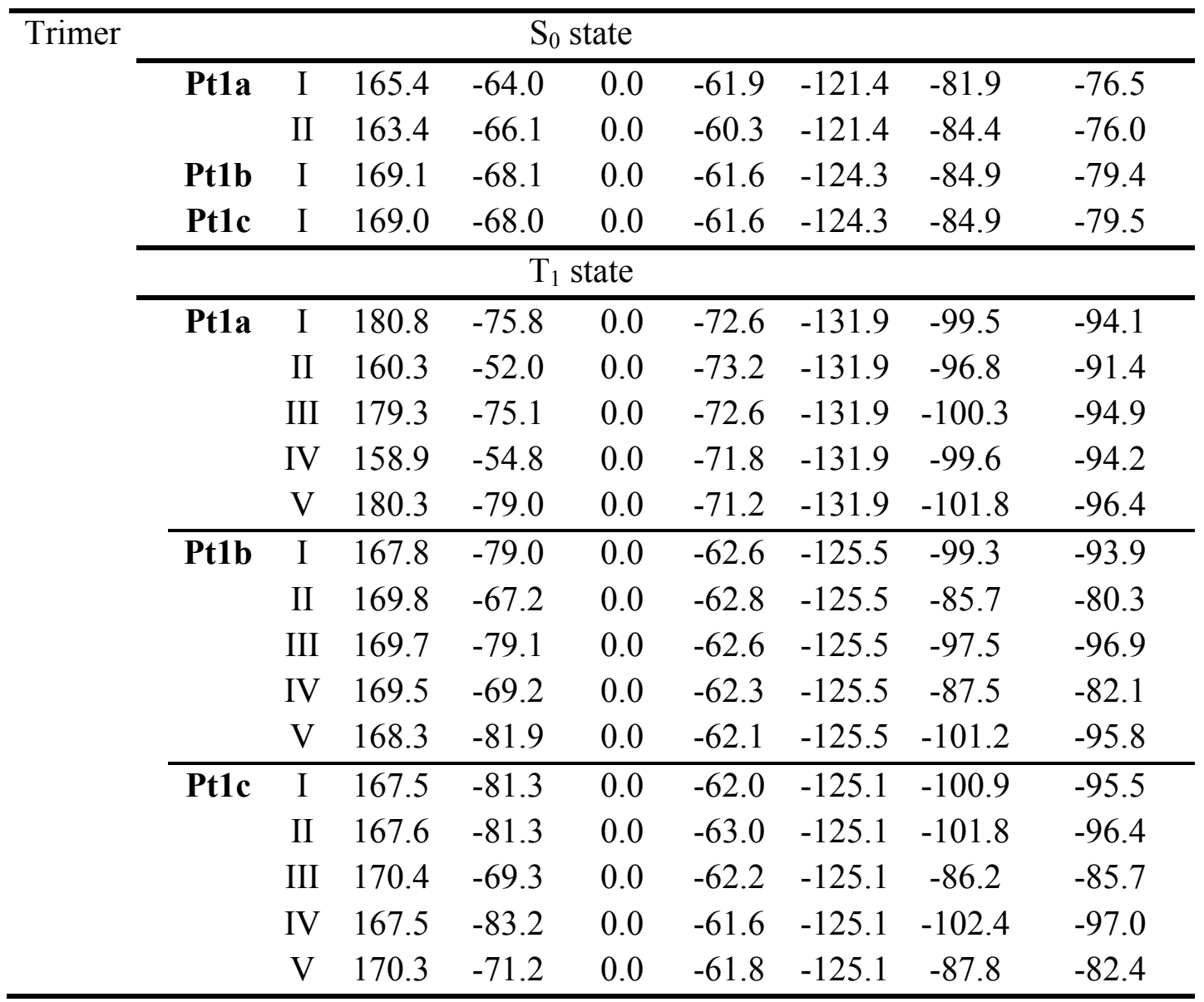

We now analyze the origins of the extra energetic stabilization when going from dimer to trimer excimers of Pt1a. Type I and type III fragmentations of dimer and trimer Pt1a, respectively, are used to scan the potential energy curve of trimer Pt1a along the varying Pt $\cdots \mathrm{Pt}$ bond distances (see below). The curve was built up by simultaneously elongating the two Pt $\cdots$ Pt bond distances from 2.9 to $5.7 \AA$ in both the $\mathrm{S}_{0}$ and $\mathrm{T}_{1}$ states. The corresponding EDA $\Delta E_{\text {tot }}$ curves are illustrated in Figure 5a-b, while the individual energy contributions to $\Delta E_{\text {tot }}$ are displayed in Figure 5c-f. The first thing to note is that the potential energy profile for the dimer of Pt1a is analogous for both the ground (aggregate) and triplet (excimer) states, displaying minima around an interlayer distance of ca. 3.4-3.5 $\AA$ (see Figure 4a), which is in very good agreement with the interlayer distances obtained in their optimized geometries (we recall to Figure 2). Furthermore, the contributions to the $\Delta E_{\text {tot }}$ are quite the same for the $\mathrm{S}_{0}$ and $\mathrm{T}_{1}$ states (compare Figure $5 \mathrm{c}$ and Figure $5 \mathrm{e}$ ). Conversely, the $\mathrm{S}_{0}$ and $\mathrm{T}_{1}$ potential energy profiles for the Pt1a trimer are very different (see Figure 5b). Remarkably, while the $\mathrm{S}_{0}$ potential energy curve possesses one single minimum, the $T_{1}$ potential energy curve is more shallow and its minimum is displaced to smaller interlayer distances, in agreement with the fact that different conformers displaying very different interlayer distances (i.e., Pt1a and Pt1b) are energetically close-lying. 
The EDA reveals that the contributions to the $\Delta E_{\text {tot }}$ are different for the $\mathrm{S}_{0}$ and $\mathrm{T}_{1}$ states (compare Figure 5d and Figure 5f). A simple comparison between the latter two figures reveals that the $\Delta E_{\text {ele }}$ term sharply increases with decreasing Pt $\cdots \mathrm{Pt}$ bond distance(s) in the case of $\mathrm{S}_{0}$ (Figure $5 \mathrm{~d}$ ) but this increase is not so remarkable for $\mathrm{T}_{1}$ (Figure 5f). In addition, the $\Delta E_{\text {exrep }}$ and $\Delta E_{\text {corr }}$ contributions also differ. One can thus conclude that the decreased electrostatic repulsion in the Pt1a excimer leads to the cooperative effects observed when going from dimer to trimer excimers. These changes undoubtedly root on the feasibility of delocalizing the spin density within the three molecular units on the $\mathrm{T}_{1}$ state of the Pt1a trimer through the Pt $\cdots \mathrm{Pt}$ contacts (see Figure 3a), something neither observed on the other trimer conformers (Pt1b and Pt1c) nor on the dimer states. The decreased electrostatic repulsion in the triplet state for the trimer excimer of Pt1a (as compared to the trimer aggregate) can clearly be seen in the electrostatic potential maps, which are found in Figure S6.

\section{Dimer Pt1a}
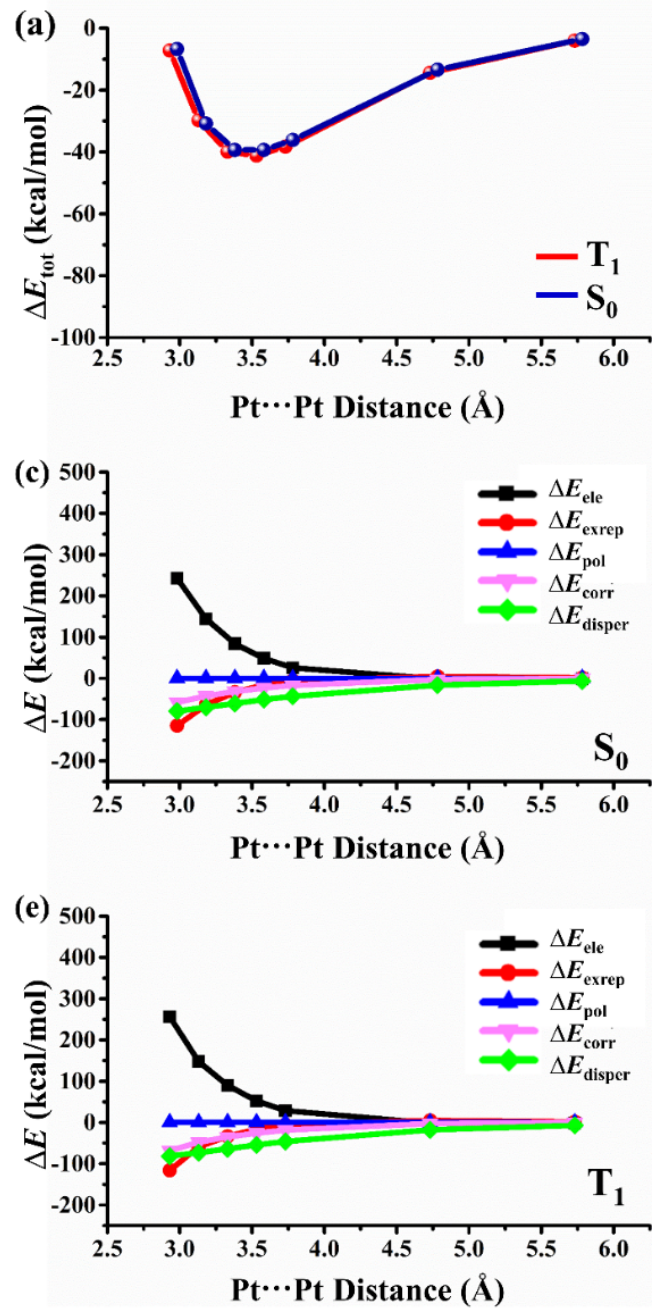

Trimer Pt1a
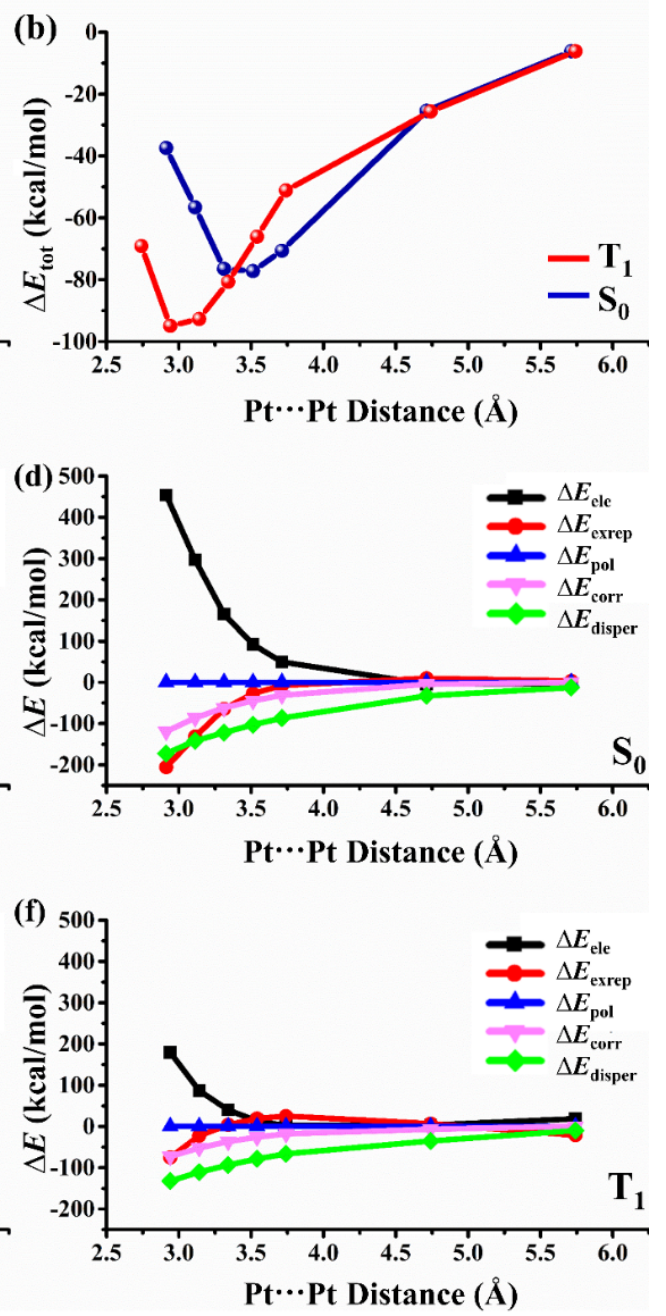
Figure 5. Potential energy curves B3LYP-D3/def2-TZVP of dimer and trimer aggregates and excimers of Pt1a along with their Pt $\cdots$ Pt distances (a) total interaction energies with BSSE correction of dimer conformer of Pt1a in $\mathrm{S}_{0}$ and $\mathrm{T}_{1}$ states and (b) total interaction energies of trimer conformer of Pt1a in $\mathrm{S}_{0}$ and $\mathrm{T}_{1}$ states. GKS-EDA results in $\mathrm{S}_{0}$ state for (c) dimer conformer of Pt1a and (d) trimer conformer of Pt1a. GKS-EDA results in $\mathrm{T}_{1}$ state for (e) dimer conformer of Pt1a and (f) trimer conformer of Pt1a. For trimer aggregates and excimers Pt1a, both $\mathrm{Pt}_{\mathrm{A}} \cdots \mathrm{Pt}_{\mathrm{B}}$ and $\mathrm{Pt}_{\mathrm{B}} \cdots \mathrm{Pt}_{\mathrm{C}}$ were symmetrically distorted to build up the potential energy curves.

\section{CONCLUSIONS}

In summary, in this work we stress the importance of synergistic effects between noncovalent interactions in isoquinolinyl pyrazolate Pt(II) complexes. Two main intermolecular forces drive the formation of aggregates and excimers: intermolecular $\pi \cdots \pi$ stacking interactions and $\mathrm{Pt} \cdots \mathrm{Pt}$ contacts. The cooperativity effects between non-covalent interactions drive the formation of trimer excimers through strengthened metallophilic Pt $\cdots$ Pt contacts; which are otherwise neither present in the dimer excimers nor in any of the ground state aggregates herein investigated. The synergistic effects are associated with a striking change in the photophysical properties when moving from very diluted solutions to concentrated dilutions or thin films, i.e., yielding red shifted-emission bands and larger photoluminescence quantum yields in the films, as we reported experimentally in our previous contribution. ${ }^{59}$ The latter properties are beneficial towards attaining very efficient near infrared organic-light emitting diodes (OLEDs). We have performed DFT calculations in monomers, dimers and trimers aggregates and excimers to rationalize the cooperativity effects and the changes in the photophysical properties. Already the computed geometric features at some of the trimer excimers point to strong cooperativity effects when going from dimers to trimers, i.e., the interlayer distances are shortened by ca. $0.5 \AA$ in some cases. These results agree with the computed synergetic and genuine non-additive energies. In addition, EDA calculations were performed to unravel the origins of the cooperative effects. The EDA analysis reveals that while dispersion contributions are mainly responsible for the binding energies, the subtle changes in the $\Delta E_{\text {ele }}$ contributions are responsible for the cooperative effects. These changes root on the feasibility of delocalizing the spin density in the trimers through the Pt $\cdots$ Pt contacts. All in all, in this work we provide some fundamental understanding on rationalizing excimer formation in isoquinolinyl pyrazolate $\mathrm{Pt}(\mathrm{II})$ complexes. Future work should be devoted to study other 
square-planar Pt(II) architectures. The formation of these excimers and thereto their photophysical properties can be fine-tuned by the supramolecular arrangement or by subtle changes in the environment. Thus, exerting some control on the metallophilic interactions is highly desired to attain tailored molecular design strategies for optoelectronic materials. 


\section{ASSOCIATED CONTENT}

Supporting Information. Computational results for the rest of complexes (Pt2-Pt4) and cartesian coordinates for all equilibrium geometries of Pt1-Pt4 (Table S1-S6, Figure S1-S4) (PDF).

\section{AUTHOR INFORMATION}

\section{Corresponding Authors}

*E-mail: naweekung@gmail.com -Tel.+66 53-943341 ext. 126.

*E-mail: daniel.escudero@kuleuven.be -Tel. +32 16193353

\section{Note}

There are no conflicts of interest to declare.

\section{ACKNOWLEDGMENTS}

The authors wish to thank Thailand Research Fund (RSA6180044) for financially support. C. Sukpattanacharoen would like to thank The Science Achievement Scholarship of Thailand (SAST) for Ph.D. fellowship. D. Escudero acknowledges funding from Internal Funds KU Leuven. N. Kungwan would like to thank the Center of Excellence in Materials Science and Technology, Chiang Mai University, and Office of National Higher Education Science Research and Innovation Policy Council (NXPO) for partially support. Department of Chemistry, Faculty of Science, and Graduate School, Chiang Mai University, Chiang Mai, Thailand are also acknowledged.

\section{REFERENCES}

1. Schalley, C. A., Analytical Methods in Supramolecular Chemistry. Wiley: 2012.

2. Mahadevi, A. S.; Sastry., G. N., Cation- $\pi$ interaction: Its role and relevance in chemistry, biology, and material science. Chem. Rev. 2013, 113 (3), 2100-2138.

3. Ma, J. C.; Dougherty, D. A., The cation $-\pi$ interaction. Chem. Rev. 1997, 97 (5), $1303-$ 1324.

4. Reddy, A. S.; Sastry, G. N., Cation $[\mathrm{M}=\mathrm{H}+, \mathrm{Li}+, \mathrm{Na}+, \mathrm{K}+, \mathrm{Ca} 2+, \mathrm{Mg} 2+, \mathrm{NH} 4+$, and NMe4+] Interactions with the Aromatic Motifs of Naturally Occurring Amino Acids: A Theoretical Study. J. Phys. Chem. A 2005, 109 (39), 8893-8903. 
5. Reddy, A. S.; Sastry, G. M.; Sastry, G. N., Cation-aromatic database. Proteins 2007, 67 (4), 1179-1184.

6. Staffilani, M.; Hancock, K. S. B.; Steed, J. W.; Holman, K. T.; Atwood, J. L.; Juneja, R. K.; Burkhalter, R. S., Anion binding within the cavity of $\pi$-metalated calixarenes. $J$. Am. Chem. Soc. 1997, 119 (27), 6324-6335.

7. Fairchild, R. M.; Holman, K. T., Selective anion encapsulation by a metalated cryptophane with a $\pi$-acidic interior. J. Am. Chem. Soc. 2005, 127 (47), 16364-16365.

8. Schottel, B. L.; Chifotides, H. T.; Dunbar, K. R., Anion- $\pi$ interactions. Chem. Soc. Rev. 2008, $37(1), 68-83$.

9. Frontera, A.; Gamez, P.; Mascal, M.; Mooibroek, T. J.; Reedijk, J., Putting anion- $\pi$ interactions into perspective. Angew. Chem. 2011, 50 (41), 9564-9583.

10. Lu, Y.; Liu, Y.; Li, H.; Zhu, X.; Liu, H.; Zhu, W., Energetic effects between halogen bonds and anion- $\pi$ or lone pair- $\pi$ interactions: a theoretical study. J. Phys. Chem. A 2012, 116 (10), 2591-2597.

11. Kim, K. S.; Tarakeshwar, P.; Lee, J. Y., Molecular clusters of $\pi$-systems: theoretical studies of structures, spectra, and origin of interaction energies. Chem. Rev. 2000, 100 (11), 4145-4186.

12. Martinez, C. R.; lverson B. L., Rethinking the term "pi-stacking". Chem. Sci. 2012, 3 (7), 2191-2201.

13. Katz, M. J.; Sakai, K.; Leznoff, D. B., The use of aurophilic and other metal-metal interactions as crystal engineering design elements to increase structural dimensionality. Chem. Soc. Rev. 2008, 37 (9), 1884-1895.

14. Eryazici, I.; Moorefield, C. N.; Newkome, G. R., Square-planar Pd (II), Pt (II), and Au (III) terpyridine complexes: their syntheses, physical properties, supramolecular constructs, and biomedical activities. Chem. Rev. 2008, 108 (6), 1834-1895.

15. Ku, H.-Y.; Tong, B.; Chi, Y.; Kao, H.-C.; Yeh, C.-C.; Chang, C.-H.; Lee, G.-H., Luminescent Pt (II) complexes bearing dual isoquinolinyl pyrazolates: fundamentals and applications. Dalton Trans. 2015, 44 (18), 8552-8563.

16. Sun, Y.; Yang, X.; Liu, B.; Guo, H.; Zhou, G.; Ma, W.; Wu, Z., Aggregation-induced emission triggered by the radiative-transition-switch of a cyclometallated Pt (ii) complex. $J$. Mater. Chem. C 2019, 7 (40), 12552-12559.

17. Sathish, V.; Ramdass, A.; Thanasekaran, P.; Lu, K.-L.; Rajagopal, S., Aggregationinduced phosphorescence enhancement (AIPE) based on transition metal complexes-An overview. J. Photochem. Photobiol. 2015, 23, 25-44. 
18. Ehrlich, S.; Bettinger, H. F.; Grimme, S., Dispersion- Driven Conformational Isomerism in $\sigma$ - Bonded Dimers of Larger Acenes. Angew. Chem. 2013, 52 (41), 1089210895.

19. Lucas, X.; Estarellas, C.; Escudero, D.; Frontera, A.; Quiñonero, D.; Deyà, P. M., Very long- range effects: cooperativity between anion- $\pi$ and hydrogen- bonding interactions. ChemPhysChem 2009, 10 (13), 2256-2264.

20. Frontera, A.; Quiñonero, D.; Costa, A.; Ballester, P.; Deyà, P. M., MP2 study of cooperative effects between cation $-\pi$, anion $-\pi$ and $\pi-\pi$ interactions. New J. Chem 2008, 31 (4), 556-560.

21. Garcia-Raso, A.; Albertí, F. M.; Fiol, J. J.; Tasada, A.; Barceló-Oliver, M.; Molins, E.; Escudero, D.; Frontera, A.; Quiñonero, D.; Deyà, P. M., Anion- $\pi$ Interactions in Bisadenine Derivatives: A Combined Crystallographic and Theoretical Study. Inorg. Chem. 2007, 46 (25), 10724-10735.

22. Walter, S. M.; Sarwar, M. G.; Chudzinski, M. G.; Herdtweck, E.; Lough, A. J.; Huber, S. M.; Taylor, M. S., Halogen bonding and $\pi-\pi$ interactions in the solid-state structure of a butadiynylene-linked bis (iodoperfluoroarene). CrystEngComm 2013, 15 (16), 3097-3101.

23. Vijay, D.; Sakurai, H.; Sastry, G. N., The impact of basis set superposition error on the structure of $\pi$ - $\pi$ dimers. Int. J. Quantum Chem. 2011, 111 (9), 1893-1901.

24. Santos, J.; Grimm, B.; Illescas, B. M.; Guldi, D. M.; Martín, N., Cooperativity between $\pi-\pi$ and H-bonding interactions-a supramolecular complex formed by C60 and exTTF. ChemComm 2008, (45), 5993-5995.

25. Stoll, I.; Brodbeck, R.; Neumann, B.; Stammler, H.-G.; Mattay, J., Controlling the self assembly of arene functionalised 2-aminopyrimidines by arene-perfluoroarene interaction and by silver (I) complex formation. CrystEngComm 2009, 11 (2), 306-317.

26. Fu, X.-L.; Li, J.-S.; Simpson, J., Non-covalent interactions in the crystal structure of methyl 4-hydroxy-3-nitrobenzoate. Crystals 2012, 2 (2), 669-674.

27. Degtyarenko, A. S.; Domasevitch., K. V., Cadmium (II) iodide and thiocyanate complexes adopted by polycyclic 1, 4-bis (pyridazin-4-yl) benzene: interplay of coordination and $\pi-\pi$ stacking interactions. Acta Crystallogr. C 2013, 69 (3), 219-224.

28. Vijay, D.; Sastry, G. N., The cooperativity of cation $-\pi$ and $\pi-\pi$ interactions. Chem. Phys. Lett. 2010, 485 (1), 235-242.

29. Tsuzuki, S.; Honda, K.; Uchimaru, T.; Mikami, M.; Tanabe, K., Origin of attraction and directionality of the $\pi / \pi$ interaction: model chemistry calculations of benzene dimer interaction. J. Am. Chem. Soc. 2002, 124 (1), 104-112. 
30. Antony, J.; Brüske, B.; Grimme, S., Cooperativity in noncovalent interactions of biologically relevant molecules. Phys. Chem. Chem. Phys. 2009, 11 (38), 8440-8447.

31. Alkorta, I.; Blanco, F.; Deya, P. M.; Elguero, J.; Estarellas, C.; Frontera, A.; Quinonero, D., Cooperativity in multiple unusual weak bonds. Theor. Chem. Acc. 2010, 126, $1-14$.

32. Mignon, P.; Loverix, S.; D. Proft, F.; Geerlings, P., Influence of Stacking on Hydrogen Bonding: Quantum Chemical Study on Pyridine-Benzene Model Complexes. J. Phys. Chem. A 2004, 108 (28), 6038-6044.

33. Sinnokrot, M. O.; Sherrill, C. D., High-accuracy quantum mechanical studies of $\pi^{-} \pi$ interactions in benzene dimers. J. Phys. Chem. A 2006, 110 (37), 10656-10668.

34. Tauer, T. P.; Sherrill, C. D., Beyond the benzene dimer: An investigation of the additivity of $\pi-\pi$ interactions. $J$. Phys. Chem. A 2005, 109 (46), 10475-10478.

35. Gonzalez, C.; Lim, E. C., Ab initio study of the intermolecular interactions in small benzene clusters: The equilibrium structures of trimer, tetramer, and pentamer. J. Phys. Chem. A 2001, 105 (10), 1904-1908.

36. Ye, X.; Hua, L. Z.; Wang, W.; Fan, K.; Xu, W.; Hua, Z., The parallel $\pi-\pi$ stacking: a model study with MP2 and DFT methods. Chem. Phys. Lett. 2004, 397 (1-3), 56-61.

37. Swart, M.; van der Wijst, T.; Guerra, C. F.; Bickelhaupt, F. M., $\pi-\pi$ stacking tackled with density functional theory. J. Mol. Model. 2007, 13 (12), 1245-1257.

38. Yang, J.; Waller, M. P., A Systematic Approach to Identify Cooperatively Bound Homotrimers. J. Phys. Chem. A 2013, 117 (1), 174-182.

39. Tam, A. Y.-Y.; Wong, K. M.-C.; Wang, G.; Yam, V. W.-W., Luminescent metallogels of platinum (ii) terpyridyl complexes: interplay of metal $\cdots$ metal, $\pi-\pi$ and hydrophobichydrophobic interactions on gel formation. ChemComm 2007, (20), 2028-2030.

40. Rest, C.; Mayoral, M. J.; Fucke, K.; Schellheimer, J.; Stepanenko, V.; Fernández, G., Self- Assembly and (Hydro) gelation Triggered by Cooperative $\pi-\pi$ and Unconventional $\mathrm{C}-$ H $\cdots$ X Hydrogen Bonding Interactions. Angew. Chem. 2014, 53 (3), 700-705.

41. Suponitsky, K. Y.; Masunov, A. E., Supramolecular step in design of nonlinear optical materials: effect of $\pi-\pi$ stacking aggregation on hyperpolarizability. J. Chem. Phys. 2013, 139 (9), 1-16.

42. Wang, S. F.; Yuan, Y.; Wei, Y.-C.; Chan, W.-H.; Fu, L.-W.; Su, B.-K.; Chen, I.-Y.; Chou, K.-J.; Chen, P.-T.; Hsu, H.-F.; Ko, C.-L.; Hung,W.-Y.; Lee, C.-S.; Chou, P.-T.; Chi, Y. Highly Efficient Near-Infrared Electroluminescence up to $800 \mathrm{~nm}$ Using Platinum(II) Phosphore. Adv. Funct. Mater. 2020, 30 (30), 2002173. 
43. Wei, Y.-C.; Wang, S. F.; Hu, Y.; Liao, L.-S.; Chen, D.-G.; Chang, K.-H.; Wang, C.-W.; Liu, S.-H.; Chan, W.-H.; Liao, J.-L.; Hung, W.-Y.;Wang, T.-H.; Chen, P.-T.; Hsu, H.-F.; Chi, Y.; Chou, P.-T. Overcoming the energy gap law in near-infrared OLEDs by exciton-vibration decoupling. Nat. Photon. 2020, 14, 570-577.

44. Schmidbaur, H.; Raubenheimer, H. G., Excimer and Exciplex Formation in Gold (I) Complexes Preconditioned by Aurophilic Interactions. Angew. Chem. 2020, 132 (35), 1485614881.

45. Lu, C.-W.; Wang, Y.; Chi, Y., Metal Complexes with Azolate-Functionalized Multidentate Ligands: Tactical Designs and Optoelectronic Applications. Chem. Eur. J. 2016, 22 (50), 17892-17908.

46. Chi, Y.; Tsai, H.-Y.; Chen, Y.-K., Pt(II) Complexes with Azolate-containing Bidentate Chelate: Design, Photophysics, and Application. J. Chinese Chem. Soc. 2017, 64 (6), 574-588.

47. Ganesan, P.; Hung, W.-Y.; Tso, J.-Y.; Ko, C.-L.; Wang, T.-H.; Chen, P.-T.; Hsu, H.-F.; Liu, S.-H.; Lee, G.-H.; Chou, P.-T.; Jen, A. K.-Y.; Chi, Y., Functional Pyrimidinyl Pyrazolate Pt(II) Complexes: Role of Nitrogen Atom in Tuning the Solid-State Stacking and Photophysics. Adv. Funct. Mater. 2019, 29 (26), 1900923.

48. Ko, C.-L.; Hung, W.-Y.; Chen, P.-T.; Wang, T.-H.; Hsu, H.-F.; Liao, J.-L.; Ly, K. T.; Wang, S. F.; Yu, C.-H.; Liu, S.-H.; Lee, G.-H.; Tai, W.-S.; Chou, P.-T.; Chi, Y., Versatile Pt(II) Pyrazolate Complexes: Emission Tuning via Interplay of Chelate Designs and Stacking Assemblies. ACS Appl. Mater. Interfaces 2020, 12 (14), 16679-16690.

49. Ma, B.; Li, J.; Djurovich, P. I.; Yousufuddin, M.; Bau, R.; Thompson, M. E., Synthetic control of Pt...Pt separation and photophysics of binuclear platinum complexes. $J$. Am. Chem. Soc. 2005, 127 (1), 28-29.

50. Park, G.; Kim, H.; Yang, H.; Park, K. R.; Song, I.; Oh, J. H.; Kim, C.; You, Y., Amplified circularly polarized phosphorescence from co-assemblies of platinum (II) complexes. J. Chem. Sci. 2019, 10 (5), 1294-1301.

51. Cuerva, C.; Campo, J. A.; Cano, M.; Lodeiro, C., Multi- Stimuli- Responsive Properties of Aggregation- Enhanced Emission- Active Unsymmetrical PtII Metallomesogens through Self- Assembly. Chem.: Eur. J. 2019, 25 (52), 12046-12051.

52. Pyykkö, P., Strong closed-shell interactions in inorganic chemistry. Chem. Rev. 1997, 97 (3), 597-636.

53. Díez, A. 1.; Forniés, J.; Larraz, C.; Lalinde, E.; López, J. A.; Martín, A.; Moreno, M. T.; Sicilia, V., Structural and luminescence studies on $\pi \cdots \pi$ and Pt $\cdots$ Pt interactions in 
mixed chloro-isocyanide cyclometalated platinum (II) complexes. Inorg. Chem. 2010, 49 (7), 3239-3251.

54. Sluch, I. M.; Miranda, A. J.; Elbjeirami, O.; Omary, M. A.; Slaughter, L. M., Interplay of metallophilic interactions, $\pi-\pi$ stacking, and ligand substituent effects in the structures and luminescence properties of neutral PtII and PdII aryl isocyanide complexes. Inorg. Chem. 2012, 51 (20), 10728-10746.

55. Cho, Y.-J.; Kim, S.-Y.; Son, H.-J.; Cho, D. W.; Kang, S. O., Steric effect on excimer formation in planar Pt (II) complexes. Phys. Chem. Chem. Phys. 2017, 19 (7), 5486-5494.

56. Allampally, N. K.; Strassert, C. A.; De Cola, L., Luminescent gels by self-assembling platinum complexes. Dalton Trans. 2012, 41 (42), 13132-13137.

57. Katlenok, E. A.; Haukka, M.; Levin, O. V.; Frontera, A.; Kukushkin, V. Y., Supramolecular Assembly of Metal Complexes by (Aryl) I $\cdots$ dz2 [PtII] Halogen Bond. Chem. Eur. J. 2020, 26 (34), 7692-7701.

58. Yang, X.; Guo, H.; Xu, X.; Sun, Y.; Zhou, G.; Ma, W.; Wu, Z., Enhancing Molecular Aggregations by Intermolecular Hydrogen Bonds to Develop Phosphorescent Emitters for High-Performance Near-Infrared OLEDs. Adv. Sci. 2019, 6 (7), 1-9.

59. Chen, W.-C.; Sukpattanacharoen, C.; Chan, W.-H.; Huang, C.-C.; Hsu, H.-F.; Shen, D.; Hung, W.-Y.; Kungwan, N.; Escudero, D.; Lee, C.-S.; Chi, Y., Modulation of SolidState Aggregation of Square-Planar Pt(II) Based Emitters: Enabling Highly Efficient DeepRed/Near Infrared Electroluminescence. Adv. Funct. Mater. 2020, 30 (25), 2002494.

60. Lee, C.; Yang, W.; Parr, R. G., Development of the Colle-Salvetti correlation-energy formula into a functional of the electron density. Phys. Rev. B 1988, 37 (2), 785-789.

61. Becke, A. D., Becke's three parameter hybrid method using the LYP correlation functional. Chem. Phys. Lett. 1993, 98 (492), 5648-5652.

62. Grimme, S.; Antony, J.; Ehrlich, S.; Krieg, H., A consistent and accurate ab initio parametrization of density functional dispersion correction (DFT-D) for the 94 elements $\mathrm{H}-\mathrm{Pu}$. J. Chem. Phys. 2010, 132 (15), 154104-154123.

63. Andrae, D.; Häußermann, U.; Dolg, M.; Stoll, H.; Preuß, H., Energy-adjustedab initio pseudopotentials for the second and third row transition elements. Theor. Chim. Acta 1990, 77 (2), 123-141.

64. Boys, S. F.; Bernardi, F. d., The calculation of small molecular interactions by the differences of separate total energies. Some procedures with reduced errors. Mol. Phys. 1970, $19(4), 553-566$. 
65. Frisch, M.; Trucks, G.; Schlegel, H.; Scuseria, G.; Robb, M.; Cheeseman, J.; Scalmani, G.; Barone, V.; Petersson, G.; Nakatsuji, H., Gaussian 16 revision A. 03. 2016; gaussian inc. Gaussian 16 revision A. 03., Wallingford CT 2016.

66. Hopffgarten, M. v.; Frenking, G., Energy decomposition analysis. Wiley Interdiscip. Rev. Comput. Mol. Sci. 2012, 2 (1), 43-62.

67. Tang, Z.; Jiang, Z.; Chen, H.; Su, P.; Wu, W., Energy decomposition analysis based on broken symmetry unrestricted density functional theory. J. Phys. Chem. A 2019, 151 (24), $1-13$.

68. Su, P.; Jiang, Z.; Chen, Z.; Wu, W., Energy Decomposition Scheme Based on the Generalized Kohn-Sham Scheme. J. Phys. Chem. A 2014, 118 (13), 2531-2542.

69. TURBOMOLE, Version 7.1, TURBOMOLE GmbH, Karlsruhe, Germany, . TURBOMOLE Version 7.1 GmbH, Karlsruhe, Germany, 2016.

70. Weigend, F.; Ahlrichs, R., Balanced basis sets of split valence, triple zeta valence and quadruple zeta valence quality for $\mathrm{H}$ to $\mathrm{Rn}$ : Design and assessment of accuracy. Phys. Chem. Chem. Phys. 2005, 7 (18), 3297-3305. 


\section{For Table of Contents Only.}

The cooperative effects led to shortened Pt...Pt contacts in the trimer excimers, which are responsible for the striking changes in the emission properties of these isoquinolinyl pyrazolate Pt(II) complexes.

Cooperative Effects

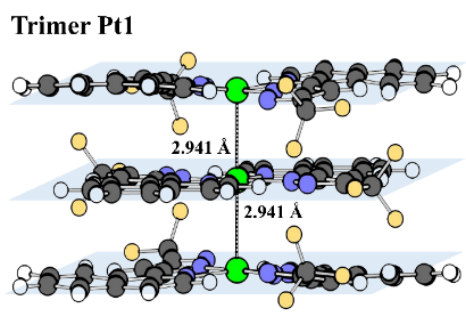

$E_{\mathrm{syn}}=-16.9 \mathrm{kcal} / \mathrm{mol} \quad E-E_{\mathrm{A}}=-8.2 \mathrm{kcal} / \mathrm{mol}$

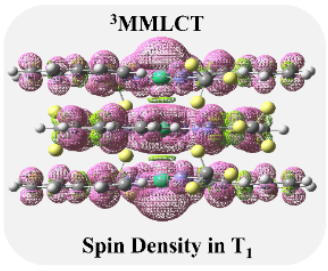

